\title{
Figurer la dystopie pour éclairer l'utopie : une fonction de l'exemple historique
}

Telling Distopia to Shed a Light on Utopia: a Rhetorical Function of Historical Example

\section{Emmanuelle Danblon}

\section{(2) OpenEdition}

Electronic version

URL: http://journals.openedition.org/aad/2111

DOI: 10.4000/aad.2111

ISSN: 1565-8961

\section{Publisher}

Université de Tel-Aviv

\section{Electronic reference}

Emmanuelle Danblon, « Figurer la dystopie pour éclairer l'utopie : une fonction de l'exemple historique », Argumentation et Analyse du Discours [Online], 16 | 2016, Online since 09 April 2016, connection on 23 September 2019. URL : http://journals.openedition.org/aad/2111 ; DOI : 10.4000/ aad. 2111

This text was automatically generated on 23 September 2019.

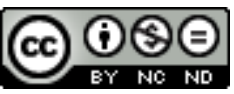

Argumentation \& analyse du discours est mis à disposition selon les termes de la licence Creative Commons Attribution - Pas d'Utilisation Commerciale - Pas de Modification 4.0 International. 


\title{
Figurer la dystopie pour éclairer l'utopie : une fonction de l'exemple historique
}

Telling Distopia to Shed a Light on Utopia: a Rhetorical Function of Historical Example

\author{
Emmanuelle Danblon
}

\section{AUTHOR'S NOTE}

Cet article a été rédigé grâce à une résidence à l'Institut d'études avancées de Paris, avec le soutien de l'État français dans le cadre du programme « Investissements d'avenir " géré par l'Agence Nationale de la Recherche (ANR-11-LABX-0027-01 Labex RFIEA+).

\footnotetext{
Alors il semble, et même il devient certain que

l'espérance est justement ce par où l'obscur

s'éclaire. L'espérance loge dans l'obscur luimême, elle participe de son opacité, de même que

l'obscur et le secret furent apparentés ; elle menace de disparaître si elle se dresse trop près, trop droit dans cet obscur.

Ernst Bloch, L'esprit de l'utopie (trad. fr. 1977, p.

Je commencerai cette contribution sur l'exemple historique en reprenant une réflexion bien connue de la rhétorique délibérative d'Aristote, à partir du passage suivant: « Denys aspire à la tyrannie puisqu'il demande une garde ; autrefois, en effet, Pisistrate, ayant ce dessein, en demandait une, et, quand il l'eut obtenue, il devint tyran » (Rhét., I, $2,1357 b)$. 
2 L'auteur de la Rhétorique étudie l'exemple (paradeigma) du point de vue de ses vertus persuasives. Il se fonde en particulier sur un topos, principe rhétorique essentiel, qui guide l'utilisation du paradeigma : "Les arguments par les fables sont plus faciles à se procurer; mais les arguments par les faits historiques sont le plus utile pour la délibération ; car le plus souvent l'avenir ressemble au passé » (Rhét. I, 2, 1394a). Ce qui va retenir mon attention ici est le caractère historique de l'exemple, source potentielle d'analogie, dans laquelle Aristote perçoit le cœur de la persuasion, avançant cet argument : « car le plus souvent l'avenir ressemble au passé ».

3 Je me pencherai plus précisément sur l'importance, dans la mémoire orale de la communauté, de l'événement historique, mémoire qui constitue dans ce cas la condition de la persuasion. C'est d'ailleurs la façon dont on peut comprendre le commentaire d'Aristote sur l'exemple historique de Denys qui aspire à la tyrannie : il y a eu d'autres cas semblables dans l'histoire mais celui de Denys se suffit à lui-même parce que tout le monde l'a bien en tête. En d'autres termes, le caractère exemplaire de l'événement historique, qui fera de lui un bon candidat au paradeigma, n'est pas fondé sur un quelconque critère de validité. Il est lié à la place qu'il occupe dans la mémoire de la communauté. Une fois ce critère " mémoriel » admis, la qualité de l'EH sera mise au service d'une décision que l'on espère utile pour la communauté. La visée argumentative au sens de Ruth Amossy (2012) est bien une décision qui sera qualifiée d'utile du fait que l'auditoire pourra effectuer une comparaison, ou mieux, une analogie, entre le cas historique et celui qui fait débat aujourd'hui. Et bien sûr nous sommes fondés à appliquer l'analogie en nous appuyant sur le topos « le plus souvent l'avenir ressemble au passé ». Il importe donc que l'événement passé soit bien présent dans la mémoire de la communauté, pour qu'il puisse offrir des éléments précis (dans le cas de Denys, le fait de demander une garde) qui se transformeront en indices, supports de l'analogie. Si l'analogie est acceptée (ce qui n'est jamais garanti), la décision coulera de source. En d'autres termes, la mémoire de la communauté portant sur un événement du passé permettra de servir de support à une décision politique, dont l'utilité s'imposera avec une force de persuasion non négligeable. Voilà pourquoi Aristote conseille à l'orateur d'utiliser l'EH, même s'il est plus rare (donc plus difficile à trouver) que l'exemple tiré de la fable.

4 Dans la suite de cette contribution, je voudrais examiner une fonction rhétorique dans laquelle l'EH joue un rôle moins souvent étudié que celui de la décision politique, directement liée à la rhétorique délibérative. Un tel rôle n'est pourtant pas sans lien avec la fonction politique du délibératif, mais elle participe aussi des fonctions de cohésion que l'on trouve dans le genre épidictique. Cette fonction qui tient à la fois du délibératif et de l'épidictique peut être identifiée comme "utopique». Elle se caractériserait par la création d'un «horizon d'attente» montrant une direction générale pour l'ensemble des décisions d'une communauté1.

5 Depuis quelques années, certains membres de l'équipe du GRAL tentent de mieux comprendre les fonctions rhétoriques contenues dans les chartes et déclarations de la modernité (Danblon et de Jonge 2006 ; Danblon 2013 ; Danblon et Mayeur 2015). Le fil de cette réflexion nous aura conduits peu à peu à dégager une possible fonction utopique contenue dans ce type de document qui participe tout à la fois du délibératif et de l'épidictique, pour le dire en termes aristotéliciens. Plus précisément, il y a clairement une visée politique dans un texte qui relate des événements matriciels pour la communauté, événements qui sont toujours des exemples historiques réels et qui sont 
généralement relatés dans les préambules. Simultanément, l'évocation de l'événement revêt une fonction pour l'avenir, mais celle-ci n'est pas directement de l'ordre de la décision politique comme elle l'est dans le genre délibératif. Nous avons proposé de rapprocher cette fonction de celle que Perelman et Olbrechts-Tyteca voient dans le genre épidictique, à savoir la création d'une disposition générale à l'action. Cette mise en disposition de l'auditoire sur le long terme, tournée vers un horizon d'attente ou un destin commun, n'est pas sans lien avec une conception technique de l'utopie défendue par Miguel Abensour ${ }^{2}(2010$; cf. aussi Danblon et Mayeur 2015).

Enfin, nous avions pu établir que le type de décision caractérisée par cette fonction utopique fonctionnait d'autant mieux qu'elle prenait appui sur un élément dystopique, événement historique présent dans la mémoire de la communauté, et servant de repoussoir aux yeux des contemporains. Je me concentrerai sur cette fonction particulière qui consiste à figurer la dystopie pour offrir ce point d'appui, levier d'une mise en disposition générale de la communauté vers un horizon d'attente. ${ }^{3}$

Dans les développements les plus récents (Danblon et Mayeur 2015), nous avions remarqué que le dispositif «dystopie - utopie » était présent dans les grandes chartes de la modernité. Il me semble maintenant que ce diptyque renvoie à une fonction plus générale (et sans doute plus ancienne) de la rhétorique des sociétés humaines. On trouve d'ailleurs de tels exemples dans l'histoire des sociétés, en particulier dans le canon biblique. Selon l'historien de l'Antiquité Ian Assmann :

La mémoire collective opère donc dans deux directions: vers l'arrière et vers l'avant. Non contente de reconstruire le passé, elle organise l'expérience du présent et de l'avenir. Aussi, serait-il absurde d'opposer un «principe mémoire » et un " principe espérance » : chacun est la condition de l'autre et n'est pas pensable sans lui (2010: 38 , je souligne).

Enfin, plus récemment, les avancées des neurosciences permettent d'affiner l'hypothèse selon laquelle la représentation du passé facilite le travail de l'imagination et de la décision pour l'avenir (Berthoz et Debru: 2015 ; Arcangeli et Docik 2015). En travaillant sur la prise de distance depuis un point de vue autocentré jusqu'à un point de vue hétéro-centré, les auteurs parviennent à objectiver certains critères du possible et du vraisemblable dans la construction de la mise en récit. Ainsi, le point de vue aristotélicien trouve de nombreuses confirmations aujourd'hui par l'éclairage de la linguistique, de l'histoire et des neurosciences.

9 Je voudrais à présent me concentrer sur la mise en récit de la dystopie, volet négatif de la mémoire de la communauté, afin d'en explorer les fonctions rhétoriques, ce qui me permettra de revenir sur le choix opéré par Assmann de la formule "principe espérance " (cf. citation supra), qui évoque, bien sûr, les réflexions sur l'Utopie d'Ernst Bloch, citées en exergue. Assmann pose en outre l'hypothèse que toute société passe en l'espace de 80 ans (deux générations) de la mémoire communicationnelle à la mémoire culturelle. En termes rhétoriques, cela a une signification notable: le passage de la mémoire communicationnelle à la mémoire culturelle s'opère par la transformation de preuves extra-techniques, comme le témoignage oral, à la preuve technique, discours oral ou écrit construit par un orateur et adressé à un auditoire spécifique (Danblon et Meyer 2015). Rappelons à ce sujet qu'Aristote range le témoignage parmi les preuves extra-techniques, le témoin oculaire représentant une "institution naturelle » selon l'heureuse expression de Renaud Dulong (1998). 


\begin{tabular}{|l|l|l|}
\hline Qualités de l'exemple historique & & \\
\hline Type de vérité & Vérité & Véracité \\
\hline \hline Test & Réalité & persuasion \\
\hline \hline Critère de vraisemblance & Empirique & psychologique \\
\hline Figure & Aphasia (énigme) & Amplification (hypotypose) \\
\hline \hline Régime cognitif & Rétention & production \\
\hline
\end{tabular}
vérité de celui de véracité. L'intérêt de l'EH est qu'il est «vrai » au sens le plus commun du terme. Son critère de vraisemblance est empirique au sens où l'on va s'appuyer sur le fait qu'il a réellement eu lieu. La figure privilégiée par le témoin qui le rapporte est de l'ordre de l'aphasia, figure qui choisit une extrême concision confinant à l'évocation, au sens de Dominicy $(2011)^{5}$. Au plan cognitif, on retrouve la tendance à la rétention dont Alain Berthoz nous dit qu'elle est la condition à toute production de sens efficace. À côté de la vérité comme critère empirique et sémantique, apparaît la véracité qui concerne le niveau du récit lui-même. Son critère de vraisemblance est psychologique. La question de la réalité empirique n'est évidemment pas incompatible avec le critère de véracité, même si celle-ci peut à l'occasion être neutralisée. Quoi qu'il en soit, l'exigence psychologique qui est à l'œuvre dans le récit demande au témoin la mise en place de techniques rhétoriques permettant de rendre vivant un événement que la communauté n'a plus forcément en mémoire. Ses figures privilégiées sont de l'ordre de l'amplification et son régime cognitif demande une production et même une profusion d'images propices à produire la persuasion. C'est en ces termes que l'on peut décrire la figure de l'hypotypose (Le Bozec 2002), sur laquelle je reviendrai.

À partir de cette distinction entre le plan de la vérité qui concerne le fait historique luimême et le plan de sa véracité qui concerne les conditions de sa mise en récit, nous pouvons à ce stade tenter de formuler une hypothèse rhétorique.

Tant qu'il existe des témoins vivants, le recours à des figures de parcimonie (énigme, ironie, allusion) est privilégié, pour laisser la place à l'effet d'évidence produit par la preuve testimoniale, extra-technique. La présence du témoin constitue avant tout une preuve extra-technique au sens d'Aristote. L'essentiel du message repose sur la présence du témoin qui atteste de l'événement comme le fait une pièce à conviction. Ceci rejoint l'intuition de Dulong qui écrit à propos du témoin oculaire que sa présence même atteste d'un «j'y étais » implicite dont l'effet d'évidence se passe en quelque 
sorte de toute technique rhétorique ${ }^{6}$. Lorsqu'il n'y a plus de témoin vivant, on passe à la mémoire culturelle, et les figures de l'amplification (cf. hypotypose) prennent le relais. Ces figures, qui confèrent une véracité à l'événement qui doit être transmis aux générations futures, sont propices à reconstruire, par la technique discursive, l'effet d'évidence, et donc de présence qui reposait principalement sur l'existence du témoin oculaire. La présence, telle que la définit Perelman ${ }^{7}$, devient alors l'effet d'une construction rhétorique. Selon le critère des Anciens, l'évidence sensible cède la place à l'évidence discursive, qui regagne la puissance persuasive par des effets entièrement construits dans et par le discours (cf. Levy et Pernot 1997).

Je voudrais à présent tester cette hypothèse sur un corpus spécifique, très éloigné du témoignage à fonction mémorielle. Il s'agit, d'une part, du préambule de la Déclaration universelle des droits de l'homme proclamée à Genève en 1948 ; d'autre part de l'exposé des motifs à la proposition de Loi dite "Taubira " pour la condamnation historique de l'esclavage et sa reconnaissance comme crime contre l'humanité. Le texte est rédigé en 1998, la loi est votée en 2001. Ces textes d'inspiration juridique ont surtout une portée éthique. Comme nous l'avons vu plus haut, cette caractéristique les rapproche à la fois du genre délibératif et du genre épidictique et participent d'une possible fonction utopique pour les communautés ${ }^{8}$.

Observons successivement la figure de l'aphasia, dans le préambule de la Déclaration Universelle des Droits de l'Homme, rédigée trois ans après la fin de l'événement historique évoqué et la figure de l'hypotypose, dans un texte rédigé plus de 200 ans après la fin de l'événement historique évoqué.

1) Extrait de la DUDH, Préambule.

Considérant que la méconnaissance et le mépris des droits de l'homme ont conduit à des actes de barbarie qui révoltent la conscience de l'humanité et que l'avènement d'un monde où les êtres humains seront libres de parler et de croire, libérés de la terreur et de la misère, a été proclamé comme la plus haute aspiration de l'homme ${ }^{9}$. 2) Loi Taubira, Exposé des motifs, 1998.

Mesdames, Messieurs, Il n'existe pas de comptabilité qui mesure l'horreur de la traite négrière et l'abomination de l'esclavage. Les cahiers des navigateurs, trafiqués, ne témoignent pas de l'ampleur des razzias, de la souffrance des enfants épuisés et effarés, du désarroi désespéré des femmes, du bouleversement accablé des hommes. Ils font silence sur la commotion qui les étourdit dans la maison des esclaves à Gorée. Ils ignorent l'effroi de l'entassement à fond de cale. Ils gomment les râles d'esclaves jetés, lestés, par-dessus bord. Ils renient les viols d'adolescentes affolées. Ils biffent les marchandages sur les marchés aux bestiaux. Ils dissimulent les assassinats protégés par le Code noir [...].Les millions de morts établissent le crime. Les traités, bulles et codes en consignent l'intention. Les licences, contrats, monopoles d'Etat en attestent l'organisation. Et ceux qui affrontèrent la barbarie absolue en emportant par-delà les mers et au-delà de l'horreur, traditions et valeurs, principes et mythes, règles et croyances, en inventant des chants, des contes, des langues, des rites, des dieux, des savoirs et des techniques sur un continent inconnu, ceux qui survécurent à la traversée apocalyptique à fond de cale, tous repères dissous, ceux dont les pulsions de vie furent si puissantes qu'elles vainquirent l'anéantissement, ceux-là sont dispensés d'avoir à démontrer leur humanité.

La France, qui fut esclavagiste avant d'être abolitionniste, patrie des droits de l'homme ternie par les ombres et les "misères des lumières", redonnera éclat et grandeur à son prestige aux yeux du monde en s'inclinant la première devant la mémoire des victimes de ce crime orphelin (je souligne). ${ }^{10}$ 
Les deux textes évoquent chacun un événement historique dont l'exemplarité remplit une visée argumentative. Cette visée est exprimée par le préambule. Dans le premier cas, les massacres de la deuxième guerre mondiale, en particulier, le génocide, même s'il n'est pas explicitement nommé, servent de "paradigme» de la barbarie. Une barbarie qui se donne comme repoussoir et finalement levier de la Déclaration des Droits de l'Homme.

En première analyse, on observe l'extrême parcimonie des moyens dans l'évocation des «actes de barbarie » dans le préambule à la Déclaration des Droits de l'Homme. Tout est évoqué, rien n'est décrit. Comme nous l'avions suggéré (Danblon et Mayeur 2015), une description détaillée de l'événement historique dans ce texte aurait sans doute produit l'effet inverse de celui recherché : la présence. Dans le second cas, la longue période de l'esclavage constitue là aussi un paradigme pour les traitements inhumains. La visée argumentative poursuivie est plus directement juridique que dans le cas de la Déclaration des Droits de l'Homme, puisqu'elle est utilisée explicitement dans un « exposé des motifs » pour un projet de loi.

19 À l'opposé encore à l'extrême parcimonie de moyens utilisés dans le préambule de la Déclaration des Droits de l'Homme, dans l'exposé des motifs à la loi Taubira, tout l'arsenal de l'amplification poétique est sollicité : les descriptions par le détail propres à l'hypotypose; les éléments qui constituent autant d'indices qui vont contribuer à la qualification de l'événement comme crime contre l'humanité.

Quoi qu'il en soit, dans les deux cas, les événements relatés correspondent à une réalité historique (en principe) non contestée. L'effort rhétorique pour poursuivre la visée argumentative ne touche donc pas à l'exigence de vérité (c'est l'affaire des juristes et des historiens), mais à l'exigence de véracité, dont les enjeux sont plus subtils à cerner.

Mon hypothèse est que cette opposition radicale dans la technique rhétorique tient en très grande partie au décalage temporel entre l'advenue de l'événement historique et sa mise en récit à des fins persuasives ${ }^{11}$. Mais le plus remarquable réside dans la présence, dans les deux cas, d'une figure qui s'apparente à l'oxymore, laquelle place en vis-à-vis deux notions opposées. Cette figure me parait correspondre parfaitement à la nécessité de mettre en récit une dystopie pour pouvoir ensuite, par contraste, figurer l'utopie, dans une déclaration ou dans une péroraison.

préambule de la Déclaration des Droits de l'Homme permet de mettre en vis-à-vis la notion de «barbarie » et celle de « conscience ». La péroraison soulignée dans l'exposé des motifs à la Loi Taubira permet de cerner le contraste entre les représentations des notions « esclavagiste » et " abolitionniste ». Ainsi, si la réalité de la mémoire humaine exige des procédés qui sont à l'opposé l'un de l'autre en fonction du décalage temporel face à l'événement historique considéré, la fonction de la dystopie comme levier de l'utopie est commune à la visée de transmission, mais aussi de création d'une disposition générale à l'action ou d'identification d'un horizon commun, dans lequel une communauté entière peut se reconnaître.

23 En conclusion, ce cas particulier nous a permis de confirmer une intuition d'Aristote. Bien sûr, il faudrait pouvoir le reproduire sur d'autres textes semblables. Mais il confirme l'idée qu'un EH attesté a plus de force persuasive qu'une fable. Il permet également d'illustrer les réflexions d'historiens, de linguistes et de neurologues sur l'importance de techniques rhétoriques propices à satisfaire le critère de véracité quand les derniers témoins vivants d'un événement attesté ont disparu. En somme, par 
la figure de l'énigme qui sert le témoignage direct par la preuve extra-technique ou par la figure de l'hypotypose qui reconstruit, par la technique rhétorique, les effets du témoignage oculaire, l'exemple historique occupe la fonction de dystopie comme levier vers la formulation d'une utopie en jetant sur elle une « obscure clarté » qui contient à la fois vigilance et espoir. Le diptyque dystopie/utopie se structure en une nouvelle figure de pensée qui n'est pas sans rappeler la figure rhétorique de l'oxymore. Cette figure de pensée rendrait hommage à l'intuition de Bloch: "l'espérance loge dans l'obscur lui-même. ». Elle permet à sa façon de mieux cerner la fonction utopique que remplit ici l'EH: cultiver la mémoire d'un événement dystopique, réputé obscur, remplit, à chaque évocation, la visée utopique d'une source d'espoir pour la communauté. Ni pleinement délibérative, ni seulement épidictique, cette disposition utopique à l'action pourrait correspondre à la fonction rhétorique que j'ai tenté de cerner dans cet article : « figurer la dystopie pour éclairer l'utopie ».

\section{BIBLIOGRAPHY}

Abensour, Miguel. 2010. L'homme est un animal utopique (Paris : Editions de la Nuit)

Amossy, Ruth \& Dominique Maingueneau. 2003. Le tournant de l'analyse du discours dans les études littéraires (Toulouse : Presses Universitaires du Mirail)

Amossy, Ruth. 2009. «Considérations théoriques », Argumentation et Analyse du Discours 2, http:// aad.revues.org/561

Amossy, Ruth. 2012. L'argumentation dans le discours (Paris : Colin)

Arcangeli, Margherita \& Jérôme Dokic. 2015. « Voyage mental dans le temps : quand l'imagination nous engage ", Alain Berthoz \& Claude Debru (éds), Anticipation et prédiction. Du geste au voyage mental (Paris : Odile Jacob), 233-246

Aristote. 1967. Rhétorique, 3 vol., traduction et commentaires par Médéric Dufour (Paris : Les Belles Lettres)

Assmann, Iann. 2010. La mémoire culturelle. Ecriture, souvenir et imaginaire politique dans les civilisations antiques (Paris : Aubier)

Berthoz, Alain \& Claude Debru (éds). 2015, Anticipation et prédiction. Du geste au voyage mental (Paris : Odile Jacob)

Bloch, Ernst. 1977 [1923]. L'esprit de l'utopie (Paris : Gallimard)

Danblon, Emmanuelle \& Emmanuel de Jonge (éds). 2006. Les droits de l'homme en discours, Argumentation et Analyse du Discours 4, http://aad.revues.org/763

Danblon, Emmanuelle Victor Ferry, Loïc Nicolas \& Benoît Sans. (éds.). 2012. Rhétoriques de l'exemple. Fonctions et pratiques (Besançon : Presses Universitaires de Franche-Comté)

Danblon, Emmanuelle. 2013. L'homme rhétorique. Culture, raison, action (Paris : Le Cerf) [Traduction italienne par Salvatore Di Piazza, L'Uomo Retorico, Mimesis, 2015] 
Danblon, Emmanuelle \& David Meyer, 2015. «À quelles conditions un témoin est-il pollinisateur? Une réflexion sur la figure de l'abeille dans le rôle du prophète ", Béatrice Fleury \& Jacques Walter (éds), Carrières de témoins de conflits contemporains, Questions de Communications 23, 87-105

Danblon, Emmanuelle \& Ingrid Mayeur. 2015. « Façonner une mémoire commune. Figures de la présence et de la communion au fondement de la dynamique utopique, Lo Sguardo, Rivista di Filosofia 17 : Tropi del pensiero : retorica e filosofia, http://www.losguardo.net

Dominicy, Marc. 2011. Poétique de l'évocation (Paris : Garnier)

Dulong, Renaud, 1999, Le témoin oculaire. Les conditions sociales de l'attestation personnelle (Paris : Editions de l'EHESS)

Ferry, Victor. 2015. Traité de rhétorique à usage des historiens (Paris : Garnier)

Le Bozec, Yves, 2002, «L'hypotypose : un essai de définition formelle », L'information grammaticale 92, 3-7

Levy, Carlos \& Laurent Pernot (éds). 1999. Dire l'évidence (Paris : L'Harmattan)

Mercier, Andrée. 2009. «La vraisemblance : état de la question historique et théorique », Temps zéro, http://tempszero.contemporain.info/document393

Perelman, Chaïm \& Lucie Olbrechts-Tyteca. 2000 [1958]. Traité de l'argumentation. La nouvelle rhétorique (Bruxelles : Editions de l'Université)

Rabatel, Alain. 2009. Homo Narrans. Pour une analyse énonciative et interactionnelle du récit (Limoges : Lambert-Lucas)

Williams, Bernard. 2006. Vérité et véracité. Un essai de généalogie (Paris : Gallimard)

\section{NOTES}

1. On trouvera le détail de la description de la fonction utopique dans Danblon et Mayeur 2015.

2. Selon Abensour, en effet, la notion d'utopie n'a de sens que dans une conception pratique de la vie publique. Ce point de vue est parfaitement compatible avec la conception aristotélicienne de la rhétorique défendue ici, qui ne s'intéresse qu'aux affaires humaines et qui ne prétend pas construire de système philosophique.

3. Voir aussi Rabatel 2009 ; Danblon 2013 : 103-126).

4. Tour l'intérêt de la réflexion philosophique de Williams consiste à proposer de transcender une opposition stérile entre les partisans d'une conception trop positiviste de la vérité et les partisans d'une conception relativiste de la vérité. Proposant de conserver l'importance des deux fonctions, il montre, comment, dans une conception généalogiste de ces notions s'instaure une continuité plus qu'une concurrence. Cette conception des choses est parfaitement compatible avec la vision généalogiste que je défends pour les liens entre rhétorique et rationalité.

5. Pour une discussion théorique, en particulier sur la notion de fonction poétique à partir de la pensée de Jakobson, on se référera en particulier aux pages 50-78.

6. Sur la notion d'institution naturelle chez Dulong, voir en particulier les pages 55-59.

7. Sur la notion de présence chez Perelman et un exposé théorique récent, je renvoie à l'excellente mise au point de Ruth Amossy (2009).

8. Bien d'autres éléments linguistiques, rhétoriques et historiques séparent ces deux textes. Pour le présent propos, je ne considérerai que l'usage de l'EH dans sa fonction dystopique.

9. http://www.un.org/fr/documents/udhr/ 
10. Pour la clarté de l'exposé, je ne garde que l'exorde et la péroraison. Le texte complet se trouve à l'adresse http://www.assemblee-nationale.fr/11/propositions/pion1297.asp.

11. Il faut bien sûr préciser que cette différence de choix rhétorique n'empêche nullement que certains EH soient évoqués par allusion au sein même d'un texte qui utilise massivement l'amplification, comme le fait l'exposé des motifs de la loi Taubira. Je remercie Victor Ferry d'avoir attiré mon attention sur ce texte. Pour une réflexion sur l'usage du paradeigma en histoire, je renvoie à son ouvrage Traité de rhétorique à usage des historiens, 2015 (Paris : Garnier).

\section{ABSTRACTS}

In this paper, dystopia is analyzed as the storytelling of a historical example in its function of guide towards utopia. Enigma and amplification as rhetorical figures are analyzed from two case studies that carry on a different link with oral memory.

Dans cet article, on analyse la dystopie comme la mise en récit de l'EH comme dans sa fonction de guide vers une utopie. Les figures de l'énigme et de l'amplification sont analysées ici à partir de deux cas particuliers qui entretiennent un rapport différent à la mémoire orale.

\section{INDEX}

Keywords: amplification, dystopia, enigma, truth, truthfulness, utopia

Mots-clés: amplification, dystopie, énigme, utopie, véracité, vérité

\section{AUTHOR}

\section{EMMANUELLE DANBLON}

Université Libre de Bruxelles 